\title{
Stochastic simulation techniques as related to innovation in communications-navigation-surveillance and air traffic management (CNS/ATM)
}

\author{
Gaspare Galati *, Maurizio Naldi, Gabriele Pavan \\ Department of Computer Science, Systems and Production Engineering (DISP), \\ Tor Vergata University, Centro "Vito Volterra", Via di Tor Vergata 110, 00133 Roma, Italy \\ Received 1 January 2002; received in revised form 7 April 2003; accepted 14 April 2003
}

\begin{abstract}
The design and operational tuning of the instruments and procedures employed in communications-navigation-surveillance (CNS) and air traffic management (ATM) often relies on stochastic simulation techniques. In this paper the application areas of simulation in the CNS/ATM context are reviewed together with the simulation methods that can help solve the main problems encountered, i.e. quick simulation techniques for the simulation of rare events, and the bootstrap technique for the evaluation of the accuracy of the results.
\end{abstract}

(c) 2003 Elsevier B.V. All rights reserved.

Keywords: Air traffic management; Air traffic control; Aircraft separation; Rare event simulation; Bootstrap; Importance sampling; Extreme value

\section{Introduction}

Both deterministic and stochastic simulation techniques are more and more used in the context of communications-navigation-surveillance (CNS) and air traffic management (ATM). The driving factors are (i) the widespread availability of powerful hardware and software tools; (ii) the trend to integrate different simulators, real

\footnotetext{
${ }^{*}$ Corresponding author.

E-mail addresses: galati@disp.uniroma2.it (G. Galati), naldi@disp.uniroma2.it (M. Naldi), pavan@ disp.uniroma2.it (G. Pavan).
} 
equipment and human elements in a network (either local or geographical); (iii) the increasing quality of models. Different breeds of simulators are employed, ranging from real-time ones (for training and operational needs) to non-real-time one (for the design and planning phases) and fast-time ones. In this paper the main simulation problems encountered in the CNS/ATM context are reviewed and appropriate simulation techniques are referred to. The relevance of fast-simulation techniques in the ATM applications is mainly related to to the need to evaluate probabilities of rare events, e.g. accidents, incidents or failures.

\section{Application areas}

Simulation is a fundamental aid to the ATM validation process, as can be seen from its decomposition into six phases proposed in [27]: (a) paper (analytical) study; (b) mock up and fast-time simulation; (c) real-time simulation; (d) shadow mode trial with prototype; (e) live mode trial with prototype; (f) pre-operational validation.

Likewise, simulation in CNS is oriented to the design, fast prototyping, and performance evaluation phases.

In the following the main areas of CNS/ATM operations where simulation is involved are reviewed.

\subsection{Safety of flights: aircraft separation and related standards}

A major issue in the safety of flights is the establishment of adequate aircraft separation standards and the fine tuning of the related procedures embodied in aircraft separation assurance systems (ASAS). Several contributions to safety endangerment due to different categories of events whose probabilities are distributed over a wide range determine target levels of safety (TLS) as low as $10^{-9}$ (mid-air collisions per flight hour) [37]. An important element is the modelling of collision risk on air routes, whose numerical solution is difficult [2].

The main challenge here is the accurate evaluation of the very low probabilities of events corresponding to stringent safety requirements.

\subsection{Weather-induced risks}

Weather phenomena (e.g. wake vortices, gust fronts, microbursts) may have a determining influence on accidents, which is now evaluated through probabilistic models in lieu of - or in addition to - the previous deterministic ones [22].

In addition to the rarity of events, an additional issue is the difficulty of modelling weather phenomena as related to flight, according to the (often inadequate) experimental data.

Wind shear is a type of air motion associated with many atmospheric processes. Aircraft statistics have shown that many accidents are due to wind shear and wind related phenomena. A general definition of wind shear refers to spatial/temporal rates of variation of wind speed and/or direction and it becomes important from 
the aviation point of view only when it is of a certain types and strengths. Wind shear may be expressed quantitatively in terms of the temporal and spatial derivatives of the wind velocity vector.

\subsection{Capacity of airports and airspace}

In the planning phase the capacity of airports and airspace has to be assessed and its relationship to flight delays has to be controlled. Several elements (e.g. gates, runways, taxiways, parkways, surface movements guidance and control systems (SMGCS) for short) have to be considered since they contribute to the overall evaluation [5].

The aim is to set load limits for ATM and for surface operations. Again, because of the difficulty of modelling many of the variables involved, massive use of empirical data (e.g. aircraft arrival rates) is done.

\subsection{Flight scheduling and route allocation}

New planning/scheduling of flights, with possible delays and detours, is needed when safety criteria are in place and the terminal area and airport capacities are reduced. The analysis of schedule modifications (e.g. move flights to off-peak hours, usage of less congested airports) to minimize delays, [23,35], is often conducted through simulation, with the goal of arriving at effective scheduling procedures. Large networks of queues are typically used as models and massive usage of empirical data is done.

\subsection{Surveillance and aircraft tracking}

The surveillance function, both in air traffic control and in SMGCS, is the main input to the control guidance and planning/routing functions, whose aim is to prevent accidents on the airport surface [12] or to predict deviations from the prescribed path in the descent path [34]. The adequacy of the surveillance function to the overall system requirements (TLS and others) can be assessed by analytical techniques in some ideal situations (e.g. widely spaced targets, lack of manoeuvres) and in most cases Montecarlo simulations are needed. Particular attention is paid to the surveillance sensors, the main one being radar [18].

\section{Advanced simulation techniques}

From the examination of the problems described in Section 2 some common threads emerge related to the simulation needs in the CNS/ATM context. The most relevant issues appear to be the following:

(a) Simulation of complex systems;

(b) Rare event simulation;

(c) Simulation in the absence of mathematical models (use of recorded data). 
The first issue is a need imposed by the ever growing complexity of the systems employed, especially when the system is actually made up of a large number of very different interacting subsystems (e.g. a network).

Examples of simulation applications to complex systems

(1) Track-while-scan algorithms with interacting multiple models, where nonlinear blocks and non-Gaussian processes have to be simulated, typically through the use of a Gaussian generator followed by the combination of a linear filter and of a zero-memory non-linearity [10].

(2) Markovian models as characterised by a complex time evolution, whose closed form solution is complicated or even intractable [11].

(3) Very large networks (10000 or more nodes), where hybrid simulation technique (i.e. the combination of analytical, equation-based, modelling for a fast and approximate steady state solution and discrete-event simulation for the detailed study of the dynamic behaviour of the network) are being proposed [20].

(4) Simulation of weather-related phenomena, in particular rain and storms, and of the weather radar signals.

Simulation of the signal received by weather radar, in its components in-phase and quadrature (I and Q), is necessary to study the signal processing and the data extraction. By means of a signal simulator it is possible to evaluate the effectiveness of signal processing algorithms in perfectly known and controlled conditions. In fact the use of real signals (i.e. acquired by a weather radar in some rain situation) does not permit such an easy and exact check with the real parameters of the phenomena.

(5) Simulation models for microburst $[1,32]$ :

Double sheet model $[16,29,30]$ is a simple dynamic model of an incompressible, irrotational flow without viscosity that can be generated by distributions of singularity points as sources and vortexes. The sources are distributed on a plane at a height $H$ from the ground such to produce a current toward the lower part of the space for a convenient horizontal extension. To assure that the ground is a surface of flow, an image distribution of singleness is inserted for $z=-H$.

Ring vortex model $[7,8,19]$ : this microburst model is based on the generation of a fluid field from a couple of ring vortexes. To describe the model closed form equations of the current flow around the ring vortex are used. The ring vortex current flow is expressed by combination of complete elliptical integrals. A downburst can be simulated using an horizontal ring vortex with radius $R_{\mathrm{V}}$ at height $H_{\mathrm{V}}$ in correspondence of which a flow of air descends along the central axis $z$ with velocity $V_{z}$.

Finite element model $[21,38]$ is a simple and realistic model again based on a stationary, irrotational and incompressible flow without viscosity. In this model the characteristics of the microburst are implemented defining an opportune potential function $\phi$ at the upper bound of the considered region. This function is distributed on a circular surface around the symmetry (usually vertical) axis. This model has three parameters: the first defines the form and the intensity of the potential distribution, the second characterises the height while the last shows the dimension of the surface where the potential function is assigned. 
For the simulation of rare events several techniques are now available, and being enhanced, aiming at the reduction of the computational cost (time) of the simulation while maintaining its accuracy. The most relevant are the following:

- Importance sampling (IS);

- Repetitive simulation trials after reaching threshold (RESTART);

- Generalized extreme value theory (GEVT).

IS and RESTART are inspired by the same basic principle: altering the process under simulation so to make rare events happen more frequently and then removing the bias so introduced by appropriately weighting the simulation results (basically the count of the number of occurrences of the event of interest).

\subsection{Importance sampling}

In IS, first applied to radar and digital communication systems [24] and more recently employed for queuing and reliability models [17], the biasing is accomplished by replacing the probability model that governs the actual phenomenon with a different model where the values of the random variable that lead to the rare event of interest are more likely to take place (Fig. 1). In simplified terms, if the rare event corresponds to the region $E$ of the sample space and the actual probability density function (pdf) of the $N$-dimensional vector variable that gathers the observations is $f(\underline{x})$, the probability of the event of interest is

$$
P=\int_{E} f(\underline{x}) \mathrm{d} \underline{x}=\int I_{E}(\underline{x}) f(\underline{x}) \mathrm{d} \underline{x},
$$

where $I_{E}(\underline{x})$ is the indicator function, being 1 if $\underline{x}=\left\{x_{1}, x_{2}, \ldots, x_{N}\right\} \in E$ and 0 elsewhere. This integral may be difficult to solve because of the particular shape of the integrand function or of the integration domain, and the estimate

$$
\widehat{P}=\frac{1}{N} \sum_{i=1}^{N} I_{E}\left(x_{i}\right)
$$

would be affected by a very large statistical error due to the rarity of the event of interest. If we alter the probability model, replacing $f(\underline{x})$ by $f^{(i s)}(\underline{x})$ (the modified or "biased" pdf), the estimator

$$
\widehat{P}^{(i s)}=\frac{1}{N} \sum_{i=1}^{N} I_{E}\left(x_{i}^{(i s)}\right),
$$

where the $x_{i}^{(i s)}$ 's are drawn from $f^{(i s)}(\underline{x})$ ) actually estimates the probability $P^{(i s)}=$ $\int_{E} f^{(i s)}(\underline{x}) \mathrm{d} \underline{x}$ that is different from $P$. But, if we multiply the simulation outcomes by the weight $w\left(x_{i}\right)=f\left(x_{i}\right) / f^{(i s)}\left(x_{i}\right)$, the resulting IS estimator $\widehat{P}_{\mathrm{IS}}=\frac{1}{N} \sum_{i=1}^{N} I_{E}\left(x_{i}^{(i s)}\right) \times$ $w\left(x_{i}^{(i s)}\right)$ is an unbiased estimator of $P$, since its expected value is: 
IMPORTANCE SAMPLING
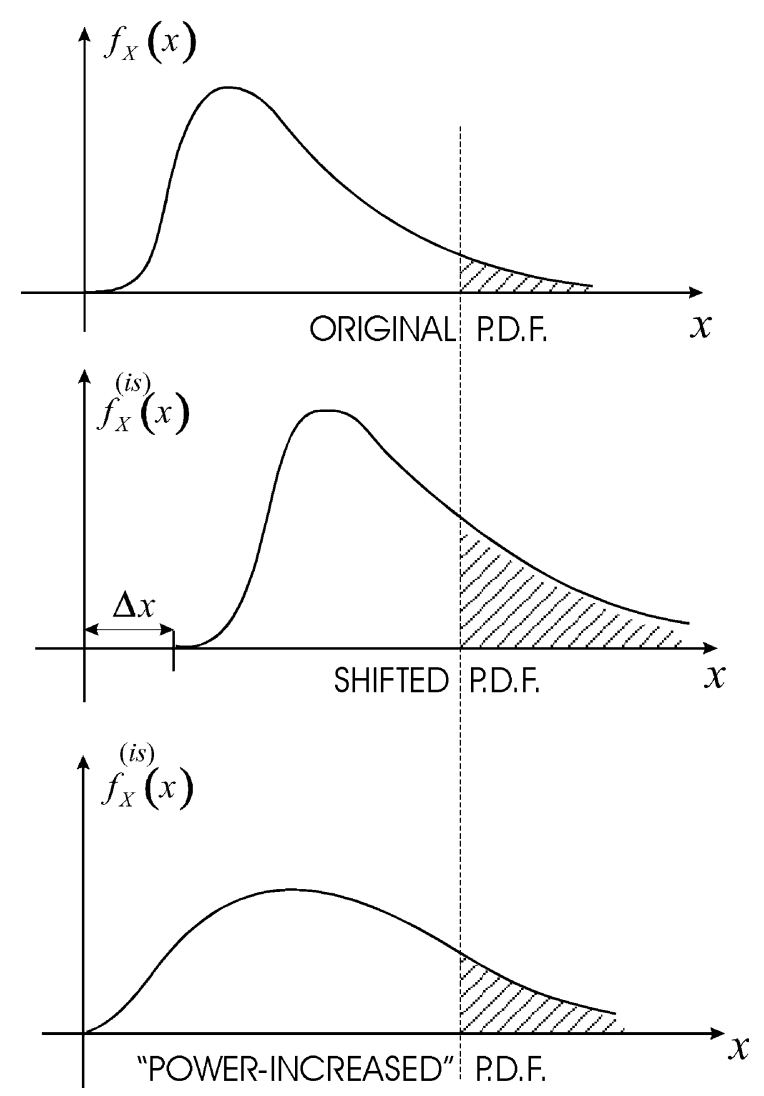

Fig. 1. IS probability density function.

$$
E\left[\widehat{P}_{\mathrm{IS}}\right]=\int I_{E}(\underline{x}) f^{(i s)}(\underline{x}) w(\underline{x}) \mathrm{d} \underline{x}=\int I_{E}(\underline{x}) f^{(i s)}(\underline{x}) f(\underline{x}) / f^{(i s)}(\underline{x}) \mathrm{d} \underline{x}=\int I_{E}(\underline{x}) f(\underline{x}) \mathrm{d} \underline{x}=P .
$$

In some cases, where the integrand function is not known, being the probability density function of the output of a complex system, we may bias the input $x$ of the system and accordingly weight the simulation outcomes (i.e. the system output $\underline{y}$ ), exploiting the fact that

$$
\int I_{E}(\underline{y}) f_{Y}(\underline{y}) \mathrm{d} \underline{y}=\int I_{E}(\underline{x}) f(\underline{x}) \mathrm{d} \underline{x}
$$

and following the above procedure.

A major problem in the implementation of IS is the proper choice of the biased function $f^{(i s)}(\underline{x})$. Common ways to construct the "biased" pdf, when problems related to the exceeding of a threshold are considered (Fig. 1), are the following: 
- Shift: $\underline{x}^{i s}=\underline{x}+\Delta \underline{x}, \Delta \underline{x} \gg \underline{0}$

- Power increase: $\underline{x}^{i s}=k \underline{x}, k>0$.

Recent advances in IS concern the adaptive choice of this function, so to arrive at the rare event probability estimation through an iterated process [31].

\subsection{Restart technique}

The RESTART technique, born in the context of high-speed communications networks [33] is based on the assumption that the state space path leading the process to the rare event (the target state) passes through some intermediate states, more frequent than the target state. Hence, when the system passes through these intermediate states, the process is altered by triggering the splitting of the trajectory: the current system state is saved and a number of independent system sub-trajectories (restarts) are simulated from that state. For example, in Fig. 2 the target state is represented by the crossing of the threshold $\mathrm{H}$, while the intermediate state is represented by the crossing of the threshold $\mathrm{L}$; the subtrajectories are the paths $B_{1} D_{11}, B_{1} D_{12}, \ldots, B_{1} D_{1 R}$. As reaching the target state is easier when starting from the intermediate states, the probability of the rare event is so artificially increased. The bias is removed by taking into account the number of restarts generated at each intermediate state.

\subsection{Generalised extreme value theory (GEVT)}

In the GEVT technique, applied first to digital communication and radar systems [36], [14] and then to queuing systems [3], the estimation of the probability of rare events is based upon the extrapolation of the tail of the probability distribution of the random variable of interest. This is done by dividing the $N$ observations in $m$ groups and considering the greatest of the $n=N / m$ elements in each group, leading

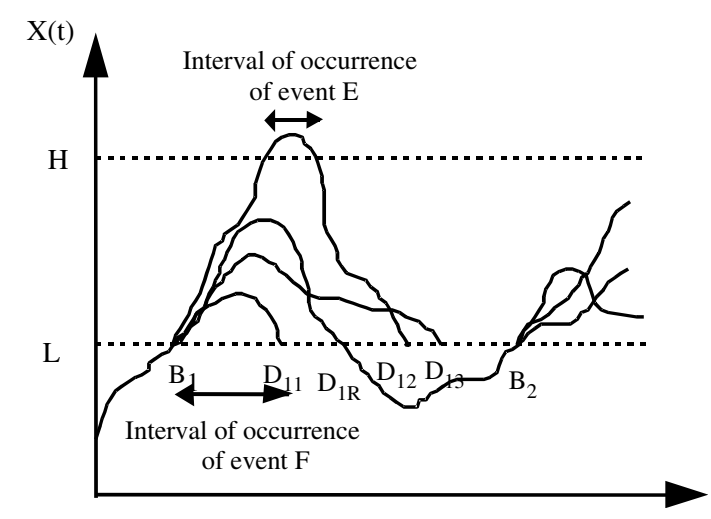

Fig. 2. Trajectories in a RESTART simulation. 
to $m$ maxima. According to Gumbel's extreme value theory [15], the distribution of maxima in a sample may converge, when $n$ increases, to one of three possible asymptotes or not converge at all. We can therefore estimate the tail of this cumulative distribution function (cdf) if we are able to tell the kind of asymptote (which is done by assumption) and to assign proper values to its parameters (which is done by an estimation procedure). By exploiting the relationship between the cdf of the maximum element in a group and the cdf common to all the elements of the group among which the maximum has been extracted, we can arrive at the cdf of the random variable of interest.

As the shape of the extreme value cdf tail is assumed to be known, the GEVT is essentially a parametric technique; this is the reason for its strength with respect to traditional simulation methods which are basically counting techniques. A consequence of this feature is that the output of GEVT is not a point estimate, but a closed form expression of the probability tail. The GEVT working hypothesis is that the probability distribution of the random variable of interest belongs to the exponential class (which includes the normal, log-normal, gamma, and $K$ distributions, beside the exponential one). A comparison of GEVT and IS is contained in [9].

A brief description of EVT and GEVT follows.

\subsubsection{Extreme value theory (EVT)}

If $X_{1}, X_{2}, \ldots, X_{n}$ are $n$ independent random variables with common distribution $F_{X}(x)$ and $Y_{n}=\max \left(X_{1}, X_{2}, \ldots, X_{n}\right)$, the cdf of $Y_{n}$ is: $F_{Y_{n}}(y)=\left[F_{X}(y)\right]^{n}$. The EVT considers the form of $F_{Y_{n}}(y)$ as $n \rightarrow \infty$. Since the initial distribution $F_{X}(x)$ is sometimes unavailable, the aim is to examine whether there is an unique distributions for $F_{Y_{n}}(y)$ when $n \rightarrow \infty$ and independent of the form of $F_{X}(x)$.

This is not unlike looking for results similar to the central limit theorem.

While the distribution function $F_{Y_{n}}(y)$ becomes increasingly insensitive to the exact distributional features of $X$ as $n \rightarrow \infty$ no unique results can be obtained which are completely independent of the form of $F_{X}(x)$. Some features of the distribution $F_{X}(x)$ are important and the asymptotic forms of $F_{Y_{n}}(y)$ (and of the distribution of the minima $\left.F_{Z_{n}}(z)\right)$ are classified into three types based on general features in the distribution tails of $X$.

Consider the Gumbel's type I asymptotic distribution of maximum values. It is the limiting distribution of $Y_{n}$ (as $\left.n \rightarrow \infty\right)$ from an initial distribution $F_{X}(x)$ whose right tail is unbounded and of an exponential type, that is, $F_{X}(x)$ approaches the unity at least as fast as an exponential distribution. For this case, we can express $F_{X}(x)$ in the form $F_{X}(x)=1-\mathrm{e}^{-g(x)}$ where $g(x)$ is an increasing function of $x$. A number of important distributions fall into this category, such as Normal, Lognormal, Rayleigh and Gamma distributions. Denoting by $F_{Y}(y)$ the asymptotic distribution of the maxima, i.e. $\lim F_{Y_{n}}(y)=F_{Y}(y)$ (in other words, let $Y_{n}$ converge to $Y$ in distribution), we have the following important result.

Theorem 1 ([13,15]). Let the r.v.'s $X_{1}, X_{2}, \ldots, X_{n}$ be independent and identically distributed with the same distribution function $F_{X}(x)$. If $F_{X}(x)=1-\mathrm{e}^{-g(x)}$, we have $F_{Y}(y)=\exp \left[-\mathrm{e}^{-\alpha(y-u)}\right]-\infty<y<\infty$ where $\alpha(>0)$ and $u$ are the scale and the loca- 
tion parameter, respectively. In particular $u$ is the mode of $Y$ (i.e. $f_{Y}(u)$ is the maximum value of the probability density function of $Y$ ), the mean of $Y$ is $m_{Y}=u+\frac{\gamma}{\alpha}$, where $\gamma \cong 0.577$ is the Euler constant, and its variance is given by $\sigma_{Y}^{2}=\frac{\pi^{2}}{6 \alpha^{2}}$.

\subsubsection{Generalised EVT}

Let $F_{X}(\cdot), v>0$, be of the exponential type with respect to $t^{v}: F_{X}(t)=$ $1-\exp \left[-g\left(t^{v}\right)\right]$, where $g(\cdot)$ is an increasing function; by linearizing $g(\cdot)$ around the point $a_{n}$ such that $F_{X}\left(a_{n}\right)=1-1 / n$, the cumulative distribution function of the maximum $Y_{n}$, i.e. $\left[F_{X}(\cdot)\right]^{n}$, becomes:

$$
F_{Y_{n}}\left(y_{n}\right)=\left[1-\frac{1}{n} \exp \left(-\frac{y_{n}^{v}-a_{n}^{v}}{c_{n}}\right)\right]^{n}
$$

where $c_{n}=b_{n} v a_{n}^{v-1}$ with $b_{n}$ such that

$$
F_{X}\left(a_{n}+b_{n}\right)=1-\frac{1}{n \cdot e} .
$$

Using

$$
\lim _{z \rightarrow \infty}\left(1+\frac{\alpha}{z}\right)^{z}=\exp (\alpha)
$$

we have

$$
\lim _{n \rightarrow \infty}\left[F_{X}\left(a_{n}^{v}+c_{n} y\right)^{1 / v}\right]^{n}=\exp \left\{-\mathrm{e}^{-y}\right\} .
$$

Weinstein [36] has shown that $F_{X}(\cdot)$ is an exponential type iff

$$
\lim _{n \rightarrow \infty} n\left[1-F_{X}\left(a_{n}^{v}+c_{n} y\right)^{1 / v}\right]=\exp (-y) \text {. }
$$

The GEVT approximation is based upon above expressions for a finite value of $n$; for $v=1$ we obtain the classical EVT approximation.

Posing:

$$
x=a_{n}^{v}+c_{n} y \text { and } a_{n}^{*}=a_{n}^{v},
$$

the extrapolated tail is

$$
1-F_{X}(x)=\frac{1}{n} \exp \left\{-\frac{x^{v}-a_{n}^{*}}{c_{n}}\right\}
$$

and the approximated distribution of the maxima is

$$
F_{2}(x)=\exp \left[-\exp \left(-\frac{x^{v}-a_{n}^{*}}{c_{n}}\right)\right]
$$

the parameter $v$ allow to extend the domain where the approximation is fairly good [with respect to the EVT (i.e. to $v=1$ )].

From a given sample and the approximated distribution of the maxima, the relevant parameters are estimated. The probability of exceeding any given value $x$ (probability of false alarm, $P_{f a}$, in the radar case) is evaluated by equation 


$$
1-F_{X}(x)=\frac{1}{n} \exp \left\{-\frac{x^{v}-a_{n}^{*}}{c_{n}}\right\} .
$$

The result is exact for $X=a_{n}$ (by definition) and nearly exact in an interval around $a_{n}$, widened by introducing the parameter $v$ (GEVT).

The advantages of GEVT method are:

- valid for most probability distributions (Gaussian, Rayleigh, Gamma, Lognormal, $K$ )

- gives a closed-form expression of the probability tail : $Q(x)=1-F_{X}(x)$

- the algorithm is general (should not be adapted to the input data)

- the computational cost is acceptable.

The estimation problem is the following: given a sample: $\left(x_{1}, x_{2}, \ldots, x_{N}\right)$ of the $N$ observations, it is divided into $m$ groups (each of $n$ elements) with $N=m \cdot n$, and the $m$ maxima are determined $\left(y_{1}, y_{2}, \ldots, y_{m}\right)$; the parameters $a_{n}^{*}, c_{n}, v$ are estimated from the set $\left(Y_{1}, Y_{2}, \ldots, Y_{m}\right)$ using the approximated distribution of the maxima

$$
F_{2}(y)=\exp \left[-\exp \left(-\frac{y^{v}-a_{n}^{*}}{c_{n}}\right)\right] .
$$

The estimation of the parameter of the extrapolated tail can be done [14] by means of:

- maximum likelyhood method (ML)

- linear approximation (LA).

The performance are equivalent and the latter is considered here; it has the advantage of simplicity and is performed in the following steps:

1. The distribution of the maxima is linearized by the transformation $F_{2}^{-1}(\cdot)$

2. A linear regression model is assumed for the data $X_{i}^{v}$

3. The regression coefficients, $a_{n}^{*}$ and $c_{n}$, are estimated by the standard method (least squares)

4. The parameter $v$ is selected in order to maximise the correlation coefficient.

\subsection{Evaluation of the accuracy: the bootstrap method}

An important issue in the selection of the appropriate simulation technique is the evaluation of its computational cost in comparison with other techniques, with the goal of establishing preference domain of applicability for each technique on the basis of the knowledge of the problem at hand, the size of the system under simulation and the order of magnitude of the probability to be estimated [4,25].

A problem recurring in many of the application areas reviewed in Section 2 is that the available observations of the phenomena involved (e.g. the delays incurred or the extent of detours) are not amenable to mathematical models. As an alternative to the 
use of quite rough analytical approximations, the simulation approach can take advantage of the combination of the plug-in principle and the bootstrap method [6,26].

The plug-in principle allows us to bypass the need for a model of the probability distribution function $F$ of the random variable governing the phenomenon under investigation. If we are interested in a parameter of this distribution or in any quantity that depends on this distribution through a function $\theta=t(F)$, but we do not know $F$, we can replace the actual distribution function by its empirical counterpart $\hat{F}$ (the related density function has equal mass $n^{-1}$ at each point $x_{i}$ ) and obtain the corresponding plug-in estimate from the application $\hat{\theta}=t(\hat{F})$. As the plug-in principle solves the problem of estimating the parameter of interest when all we know about the phenomenon is the observed sample of its realisations, the bootstrap methods provides us with a straightforward evaluation of its accuracy. Given a sample $\underline{x}$ of observations $\left(x_{1}, x_{2}, \ldots, x_{m}\right)$, on which basis we have obtained a single estimate $\hat{\theta}$ of the quantity of interest, we generate $B$ bootstrap replications of $\underline{x}$ by resampling with replacement from the original sample, i.e. by the application of a random pointer to the entries of the vector gathering the observations. This is equivalent to generate $B$ sets of $m$ pseudorandom quantities each from the empirical distribution function $\hat{F}(x)$, using the well-known analytical inversion technique [28]. For each of the bootstrap replications we can obtain an estimate $\hat{\theta}_{i}^{*}(i=1,2, \ldots, B)$. Fig. 3 shows the scheme of the bootstrap method. The standard deviation of the set $\left(\hat{\theta}_{1}^{*}, \hat{\theta}_{2}^{*}, \ldots, \hat{\theta}_{B}^{*}\right)$ is a basic indication of the accuracy of the estimate. Confidence intervals can be built for $\hat{\theta}$ by exploiting (for large values of $B$ ) or by approximating the empirical distribution $\hat{F}(\hat{\theta})$.

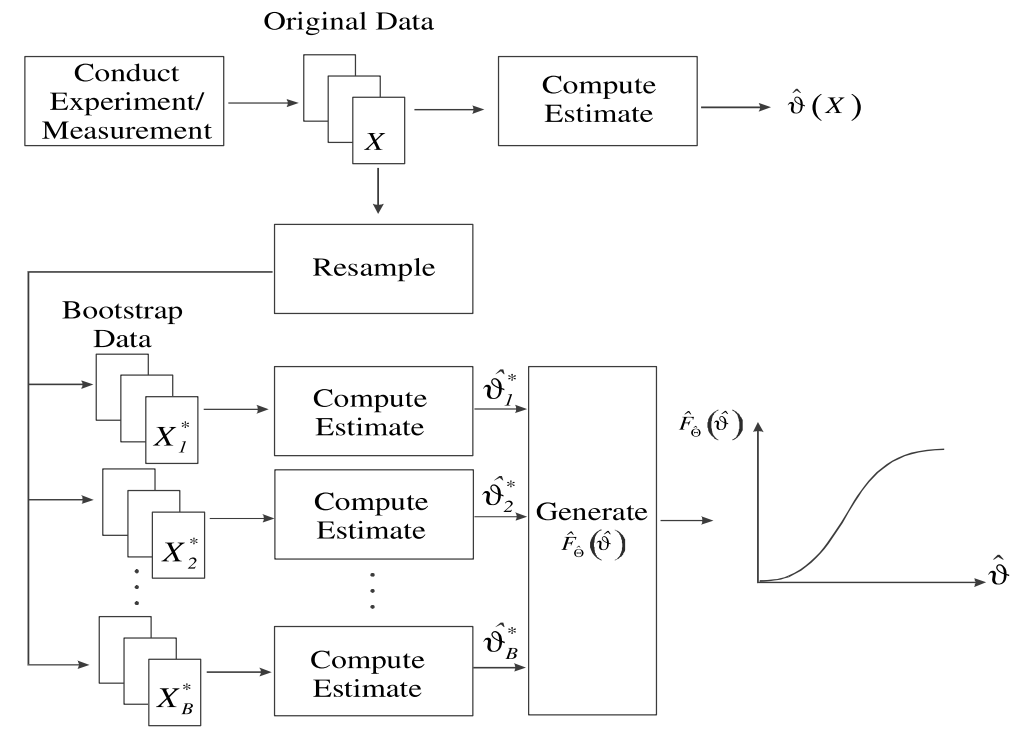

Fig. 3. Scheme of the bootstrap method. 
The bootstrap method can be used for hypotheses testing, a key element in the applications considered here as well as in many signal processing applications (e.g., in radar [18]) by generating $B$ bootstrap statistics and using the $M$ th rank statistics $(M$ being related to the level of significance: $\alpha=(B+1-M) /(B+1)$.

As compared other methods, the bootstrap is very valuable when data sizes are too small to use asymptotic result.

\section{Conclusions}

The main application areas of simulation in the context of CNS and ATM have been reviewed. The most relevant challenges posed to the simulation techniques in this context appear to be the simulation of complex systems, the simulation of rare events, and the simulation in the absence of mathematical models (where recorded data can be used instead). Three techniques, namely IS, GEVT and RESTART, have been described for the quick simulation of rare events. The combination of the plugin principle and of the bootstrap method has been suggested as a tool to bypass the lack of appropriate mathematical models.

\section{References}

[1] M.R. Babcock, K.K. Droegemeier, Numerical simulation of microburst: aircraft trajectory studies, Preprints, in: 3rd Conference on the Aviation Weather System, American Meteorological Society, Boston, 1989, pp. 62-67.

[2] G.J. Bakker, H.A.P. Blom, Air traffic collision risk modelling, in: Proceedings of the 32nd IEEE Conference on Decision and Control, 1993, pp. 1464-1469.

[3] F. Bernabei, R. Ferretti, M. Listanti, G. Zingrillo, A methodology for buffer design in ATM switches, European Trans. Telecomm. 2/4 (1991) 367-379.

[4] G. D'Acquisto, M. Naldi, Computational costs of fast stochastic simulation techniques for Markovian fluid models in multiservice networks, Simulation Practice and Theory 9 (2001) 255-272.

[5] G.L. Donohue, W.D. Laska, United States and European airport capacity assessment using the GMU macroscopic capacity model, in: 3rd USA/Europe Air Traffic Management R \& D Seminar, Napoli, 2000.

[6] B. Efron, R.J. Tibshirani, An Introduction to the Bootstrap, Chapman \& Hall, 1994.

[7] W. Frost, R.L. Bowles, Wind shear terms in the equations of aircraft motion, J. Aircraft 21 (11) (1984).

[8] W. Frost, Flight in low-level wind shear, NASA CR 3678 (March) (1983).

[9] G. Galati, A. Di Vito, D. Iovino, An overview of computer simulation techniques for signal processing and system analysis, in: European Simulation Multiconference, Roma, 1989.

[10] G. Galati, R. Vernini, Tracking of manoeuvring targets using the orientation angle, in: International Conference on Microwaves and Radar MIKON 98, Poland, Workshop 1, 1998.

[11] G. Galati, R. Dellago, F. Franco, Advantages of ground-based integration facilities for GNSS: the landing radar (Lara) case, in: International Conference on Radar Systems, Brest, 1999.

[12] G. Galati, F. Marti, F. Rocci, Generation of radar image of aircraft for design and test of image processing algorithms in radar surveillance applications, in: Regazzoni, Fabri, Vernazza (Eds.), Advanced Video-Based Surveillance Systems, Kluwer, 1999.

[13] B. Gnedenko, Sur la distribution limite du terme maximum d'une série aléatoire, Ann. Math. 44 (1943) 423-453. 
[14] M. Guida, D. Iovino, M. Longo, An empirical study of EVT and GEVT estimates of probability tails for radar applications, in: Proceedings of the CIE International Conference on Radar, Nanjing, 1986.

[15] E.J. Gumbel, Statistics of Extreme, Columbia Univ. Press, New York, 1958.

[16] K.U. Hahn, Takeoff and landing in a downburst, J. Aircraft 24 (8) (1987).

[17] P. Heidelberger, Fast simulation of rare events in queuing and reliability models, ACM Trans. Model. Comput. Simul. 5/1 (1995).

[18] IEE Proceedings, Radar, sonar and navigation, Modelling and Simulation of Radar Systems 148(3) (2001) (special issue).

[19] M. Ivan, A. Ring, Vortex downburst model for flight simulations, J. Aircraft 23 (3) (1986).

[20] G. Kaplan, Simulating networks, IEEE Spectrum 38/1 (2001).

[21] H. Kardenstuncer, D.H. Norrie, Finite Element Handbook, McGraw-Hill, 1987.

[22] J. Kos, H.A.P. Blom, L.J.P. Speijker, M.B. Klompstra, G.J. Bakker, Probabilistic wake vortex induced accident risk assessment, in: 3rd USA/Europe Air Traffic Management R \& D Seminar, Napoli, 2000.

[23] P.F. Kostiuk, D. Lee, D. Long, Closed loop forecasting of air traffic demand and delay, in: 3rd USA/ Europe Air Traffic Management R \& D Seminar, Napoli, 2000.

[24] R.L. Mitchell, Importance sampling applied to simulation of false alarm statistics, IEEE Trans. Aerospace Electron. Syst. 17/1 (1981) 15-24.

[25] M. Naldi, F. Calonico, A comparison of the GEVT and RESTART techniques for the simulation of rare events in ATM networks, Simulat. Pract. Theory 6/2 (1998) 181-196.

[26] D.N. Politis, Computer-intensive methods in statistical analysis, IEEE Signal Process. Mag. 15/1 (1998) 39-55.

[27] A. Printemps, DNA experience on new ATM concepts validation, Workshop ATM '99, Capri, 1999.

[28] R.Y. Rubinstein, Simulation and the Monte Carlo Method, John Wiley, Mnaoi, 1981.

[29] G. Schanzer, Influence of wind shear on flight safety, in: AGARD-CP-347, Atene, 10-13 May, 1983.

[30] Z. Shangxiang, B. Etkin, Model of the wind field in a downburst, J. Aircraft 22 (7) (1985).

[31] P.J. Smith, M. Shafi, H. Gao, Quick simulation: a review of importance sampling techniques in communication systems, IEEE J. Selected Areas Commun. 15/4 (1997) 597-613.

[32] R.C. Srivastava, A simple model of evaporately driven downdraft: Application to microburst downdraft, J. Atmos. Science 42 (1985) 1004-1023.

[33] M. Villén-Altamirano, J. Villén-Altamirano, RESTART: a method for accelerating rare event simulations, in: 13th International Teletraffic Congress ITC13, Copenhagen, 1991.

[34] T. Warren, Trajectory prediction concepts for next generation air traffic management, in: 3rd USA/ Europe Air Traffic Management R \& D Seminar, Napoli, 2000.

[35] T.J. Weidner, S. Green, Modeling ATM automation metering conformance benefits, in: 3rd USA/ Europe Air Traffic Management R \& D Seminar, Napoli, 2000.

[36] S.B. Weinstein, Theory and application of some classical and generalized asymptotic distributions of extreme values, IEEE Trans. Inform. Theory 19/2 (1973) 148-154.

[37] A.D. Zeitlin, B. Bonnemaison, Managing criticality of ASA applications, in: 3rd USA/Europe Air Traffic Management R \& D Seminar, Napoli, 2000.

[38] O.C. Zienkiewicz, The Finite Element Method, McGraw-Hill, 1977. 